\title{
Varied Placental Pathology and Fetomaternal Outcomes: A Case Series
}

\author{
Sumitra Bachani ${ }^{1}$, Nishi Choudhary ${ }^{2}$, Ashmita Jawa $^{3}$, Anjali Dabral ${ }^{4}$, Renu Arora ${ }^{5}$
}

\begin{abstract}
The placenta plays a vital role in the fetal growth and development; and it is an important link between the mother and fetus. The placental pathology may result in hypoperfusion and fetal growth restrictions. Here we present three cases with varied placental pathologies and the effect on fetal outcome. First case is a primigravida patient with placental teratoma; in second case, a multigravida patient presented with placental chorangiosis. Third case came at 19 weeks of gestation with ultrasonography showing features of cystic changes in the placenta. The patient was followed up throughout pregnancy with serial ultrasound monitoring and terminated at 37 weeks in view of fetal growth restriction. All the three placental conditions were associated with fetal morbidities. We emphasize the need for careful sonographic examination of the placenta in the antenatal period and gross examination and histopathology of the placenta postdelivery.

Keywords: Fetomaternal outcome, Histopathology, Placenta, Ultrasonography.

Journal of South Asian Federation of Obstetrics and Gynaecology (2020): 10.5005/jp-journals-10006-1792
\end{abstract}

\section{INTRODUCTION}

The placenta plays a vital role in the fetal growth and development, and it is an important link between the mother and fetus. It enables exchange of nutrients and oxygen from the mother to the fetus and excretion of fetal waste. 'It acts as a barrier to various harmful toxins and infective organisms. The placental abnormality can have significant implications on the fetomaternal unit. ${ }^{2}$ The placental pathology may result in hypoperfusion and fetal growth restrictions. Many placental villous structural lesions and histology correlate with causes and timing of fetal growth restriction. Certain lesions confirm intrauterine fetal infections and prediction of recurrence in next pregnancy. ${ }^{3}$ It has been reported that placental pathologies can be the cause of stillbirth. ${ }^{4}$ Careful sonographic examination and gross examination of the placenta along with histopathology provide valuable information both for obstetrician and neonatologist. ${ }^{5}$ This case series presents three cases of varied placental pathology, with an aim to emphasize the need for careful sonographic examination of the placenta in the antenatal period and postdelivery with high clinical suspicion, which can aid in antenatal counseling and management.

\section{Case Descriptions}

\section{Case 1}

A 29-year-old primigravida at 32 weeks' gestation presented with suspicion of placental tumor on sonography. She had been screened in the first trimester and all reports were normal including biochemical screen, which was low-risk. Antenatal sonography at 16 weeks had detected a localized, hypoechoic complex area $(2.5 \mathrm{~cm})$ along the fetal surface of the placenta without vascularity with a thin membranous amniotic band seen separately from the placental surface. There was no evidence of gross congenital anomaly in the fetus. It was found to increase in size in subsequent scans and the band was no longer visualized. At 32 weeks of gestation, it increased to $8.5 \times 5.5 \mathrm{~cm}$, avascular on sonography (Fig. 1A). With suspicion of placental/gestational
${ }^{1-5}$ Department of Obstetrics and Gynecology, Vardhaman Mahavir Medical College and Safdarjung Hospital, New Delhi, India

Corresponding Author: Nishi Choudhary, Department of Obstetrics and Gynecology, Vardhaman Mahavir Medical College and Safdarjung Hospital, New Delhi, India, Phone: +91 9474012820, e-mail: choudharynishi@yahoo.co.in

How to cite this article: Bachani S, Choudhary N, Jawa A, et al. Varied Placental Pathology and Fetomaternal Outcomes: A Case Series. J South Asian Feder Obst Gynae 2020;12(4):245-249.

Source of support: Nil

Conflict of interest: None

trophoblastic disease, serial beta human chorionic gonadotrophin $(\beta-h C G)$ monitoring and serial ultrasound Doppler study were done. At 34 weeks of gestation, the $\beta$-hCG level was 95,486 mIU/ $\mathrm{mL}$ and ultrasound documented fetal growth restriction, fetal wt. $<5$ th centile, and placenta with grade III-IV maturity. The lesion measured $9 \times 5 \mathrm{~cm}$ complex without vascularity suggestive of a placental tumor (Table 1). She also developed obstetric cholestasis and pregnancy was terminated by the cesarean section at 37 weeks as fetus was also growth restricted with raised umbilical artery Doppler (>95th centile). She delivered a girl child of $2 \mathrm{~kg}$; $\beta$-hCG levels in the immediate postoperative period dropped to $54354 \mathrm{mIU} / \mathrm{mL}$. On gross appearance, the placenta with attached umbilical cord measured $25 \times 20 \times 3 \mathrm{~cm}$ and cord length was $30 \mathrm{~cm}$. Membranes were complete and a skin-covered globular mass measuring $11 \times 6 \times 3 \mathrm{~cm}$ was seen arising from placenta (Figs $1 \mathrm{~B}$ and $\mathrm{C}$ ). The mother and baby dyad remained healthy and were discharged. On histopathology cut section, three vessels were identified in the cord and areas of calcification were seen in the placenta. A cyst was seen measuring $3.5 \mathrm{~cm}$ in diameter with smooth surface, filled with serosanguinous fluid. Sections documented presence of a tumor showing skin along with tufts of hair follicles containing hair shafts with adnexal structures, muscle and cartilage tissue, mature adipose tissue, 

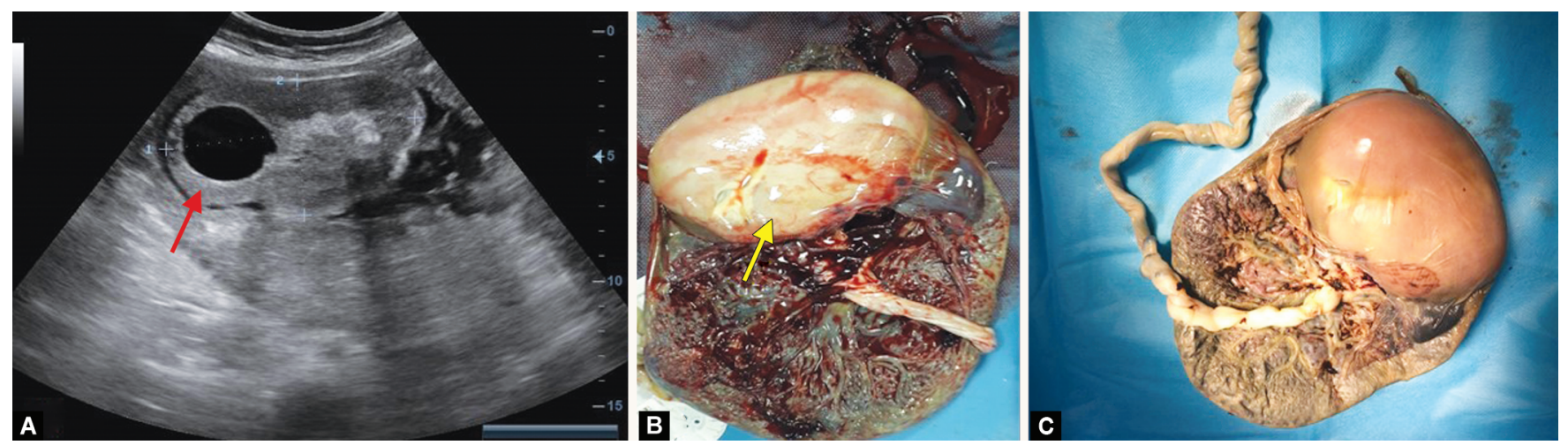

Figs 1 A to C: Placental tumor: (A) Ultrasonographic image; (B and C) Gross appearance

Table 1: Ultrasonography (USG) features at relevant period of gestation (POG) in case 1

\begin{tabular}{ll}
\hline POG & USG \\
\hline 16 weeks & A localized, complex, hypoechoic area of about \\
& $\begin{array}{l}2.5 \mathrm{~cm} \text { was noted along the fetal surface of placenta } \\
\text { without vascularity with a thin membranous } \\
\text { amniotic band seen separately from placental } \\
\text { surface with no evidence of gross congenital } \\
\text { anomaly. }\end{array}$ \\
& Single live fetus of computed gestational age \\
30 weeks & $30^{+3}$ weeks with a hypoechoic area measuring \\
& $85 \times 55 \mathrm{~mm}$ \\
33 weeks & SLF breech with fetal wt. <5th centile with FGR with \\
& placenta normal grade III-IV with hypoechoic lesion \\
& $(9 \times 5 \mathrm{~cm})$ without vascularity, Doppler: UA PI-1.3 \\
& $(>95$ th centile) MCA PI-1.44; no evidence of fetal \\
& anemia
\end{tabular}

and nerves. Histological picture was consistent with placental mature teratoma. Her $\beta$-hCG levels showed a declining trend postoperatively to normal value within 2 weeks. Postpartum sonography after 6 weeks was normal and both baby and mother are doing well.

\section{Case 2}

A 28-year-old $G_{3} P_{2} L_{1}$ at 40 weeks of gestation presented in gynecology emergency with premature rupture of membranes since 2 days associated with foul-smelling vaginal discharge. On examination, the patient was febrile (temp. $101^{\circ} \mathrm{F}$ ). Clinical examination was suggestive of chorioamnionitis with growthrestricted fetus and reduced liquor. Uterine tenderness was present with minimal greenish foul-smelling liquor on vaginal examination. Ultrasonography (USG) was suggestive of growth-restricted fetus with severe oligohydramnios, normal placenta, and no obvious gross congenital anomaly in the baby. Pregnancy was terminated by an emergency cesarean section and she delivered a baby boy of $2.3 \mathrm{~kg}$ with an Apgar score of 6,8 . The neonate was admitted in nursery for 48 hours and got discharged. The specimen of placenta with intact membranes was sent for histopathology.

On gross examination, the placenta with umbilical cord and membranes together weighed $510 \mathrm{~g}$. The size of placenta was $20 \times 16 \times 4 \mathrm{~cm}$ and the disc- both maternal and fetal surfaces grossly appeared normal with a three-vessel umbilical cord, $20 \mathrm{~cm}$ in length. On microscopy, sections from the random areas of placenta showed dysmaturity of chorionic villi displaying hypervascularity of capillary-sized vessels (Fig. 2). More than 10 capillaries per high-power field of the chorionic villi were identified. Intervillous stroma was scantily cellular. Pathological findings were consistent with chorangiosis. An USG done 6 weeks postpartum was normal.

\section{Case 3}

A 26-year-old primigravida at 19 weeks of gestation presented in the fetal medicine division with level 2 USG suggestive of cystic changes in the placenta with a normal anomaly scan (Fig. 3). She was counseled and investigated further with differential diagnosis of partial mole or placental mesenchymal dysplasia (PMD). Her $\beta-$ hCG level was $96332 \mathrm{mIU} / \mathrm{L}$ at 20 weeks and amniocentesis was done, which revealed a normal karyotype. She was monitored by serial sonography (Figs 4 and 5), which consistently showed same cystic changes in placenta till term; however, the fetus became growthrestricted. Labor was induced at 37 weeks as she also developed obstetric cholestasis. She delivered a girl of $2.2 \mathrm{~kg}$ with Apgar 8,9. Gross examination of the placenta appeared to be normal (Fig. 6). Histopathology reported placental size of $10 \times 9 \times 6.5 \mathrm{~cm}$ with attached cord measuring $40 \mathrm{~cm}$ in length. It weighed $800 \mathrm{~g}$ and seven cotyledons were identified. On serial sectioning, the cord had three vessels with few placental villi showing hydropic changes. Placental membranes were unremarkable. The mother and baby dyad remain well till date and postpartum USG at 5 weeks was normal, $\beta$-hCG declined to $<5 \mathrm{mIU} / \mathrm{L}$.

\section{Discussion}

Ultrasonographic examination of the placenta has a key role in identifying placental pathology. Ahmed et al. emphasized the role of sonography in prenatal recognition of placental tumors especially its prognostic importance in cases of placenta teratoma. ${ }^{6}$ Placenta teratoma is an extremely rare nontrophoblastic tumor of placenta with less than 30 cases reported till now in the world literature. ${ }^{7}$ It is a benign tumor composed of all three germ layers. It has been proposed that during early embryogenesis, there is an abnormal migration of germ cells from primitive gut, which are deposited in the connective tissue of the cord and in-between amnion and fetal surface of the placenta leading to a teratoma. ${ }^{8}$

Prenatal recognition of placental teratoma has important implication in prognosis. Unlike other tumors teratoma is benign; however, certain fetal conditions have been reported. ${ }^{9}$ In the present case, pregnancy had to be terminated at 37 weeks of gestation due to obstetric cholestasis and fetal growth restriction. On prenatal sonography, placental teratoma appears as the presence of tissues of varied echogenicity, such as calcification, fat, and fluid. This neoplasm must be differentiated from partial mole, 


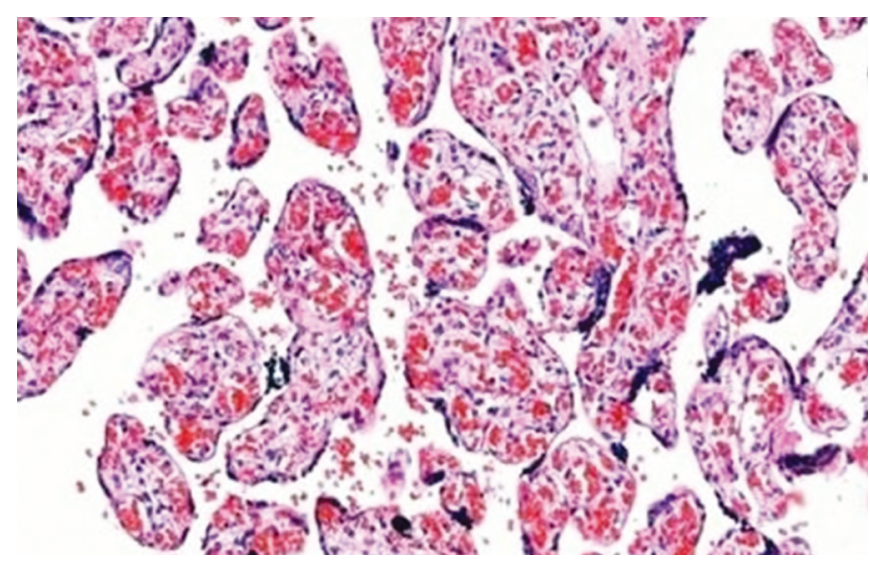

Fig. 2: Histology chorangiosis

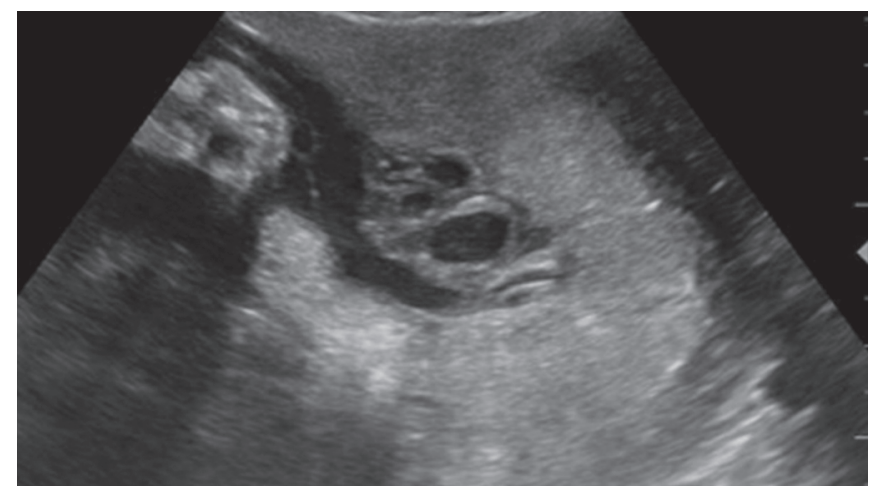

Fig. 4: Avascular, localized cystic change at 34 weeks

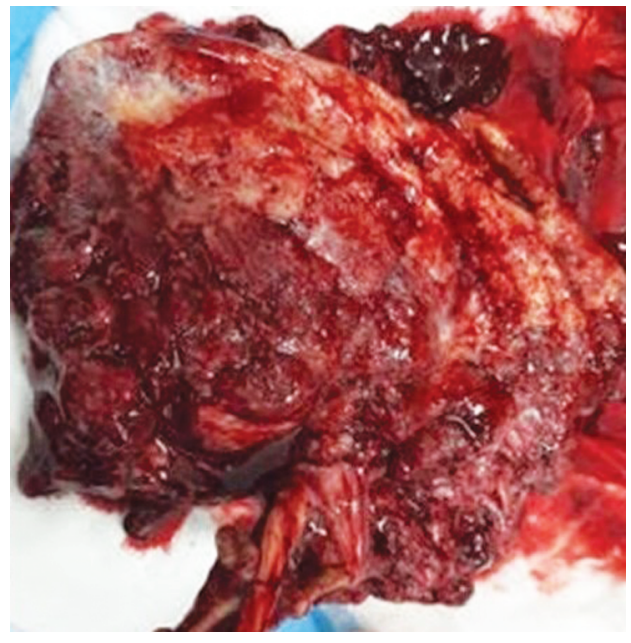

Fig. 6: Gross appearance of the placenta

complete mole with coexistent fetus, and hydropic degeneration of the placenta. In the present case, sonography revealed a hypoechoic lesion of $9 \times 5 \mathrm{~cm}$ without vascularity with raised $\beta$-hCG levels, which was later confirmed by histopathology examination. The sonographic appearance of partial mole is usually hyperechoic tissue containing numerous cystic spaces while that of complete mole usually has a separate normal placenta and in most of these cases fetus is abnormal. ${ }^{10}$ Another diagnostic challenge is to differentiate placental teratoma from fetus acardius amorphous.

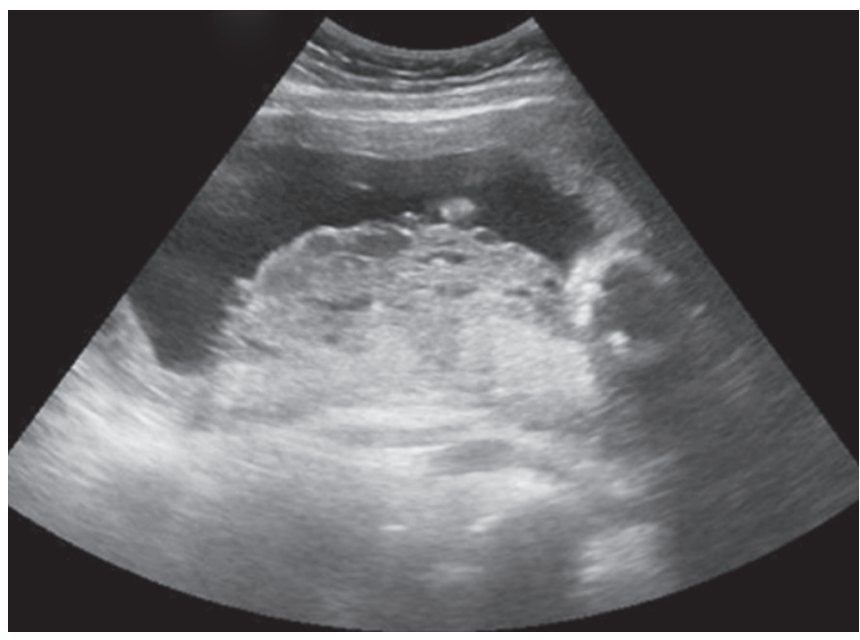

Fig. 3: Cystic changes in placenta at 19 weeks

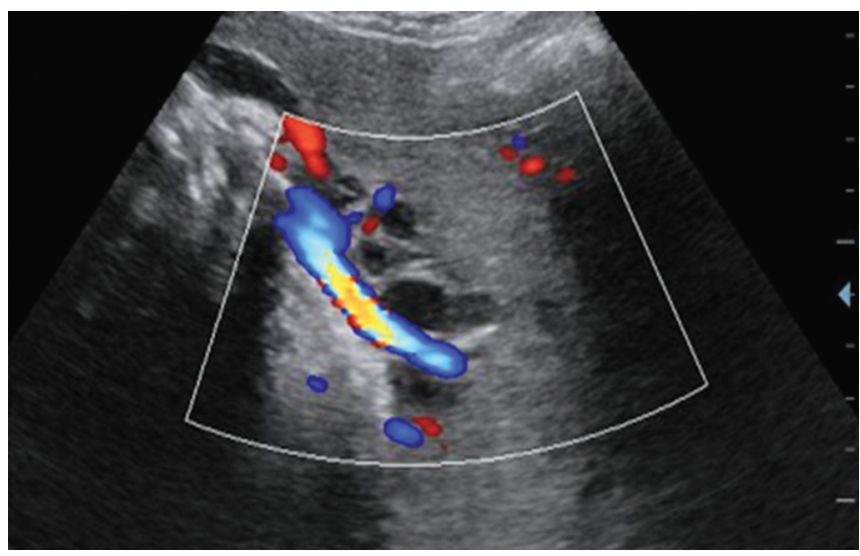

Fig. 5: Avascular, localized cystic change at 34 weeks

Ultrasonographic finding of singleton pregnancy in early scan and in addition complete lack of polarity and absence of cord-like structure aid in differential diagnosis. ${ }^{6}$ Histopathological evaluation confirms the presence of teratoma with lack of umbilical cord, absence of large feeding vessels, and totally disorganized skeletal development. ${ }^{8}$ Sex chromatin and molecular studies can further help in solving the diagnostic dilemma, though most of cases with placental teratoma have favorable prognosis.

In Case 2, chorangiosis appears to be a placental marker of antepartum hypoxia, thus aiding in correlating various clinical factors that may have led to hypoxia. Petersen et al. in their study also suggested chorangiosis as a marker of chronic hypoxia and associated with increased rate of cesarean sections for nonreassuring fetal status because of long-standing hypoxia coupled with the stress of labor. ${ }^{11}$ Chorangiosis is a rare vascular pathology of terminal chorionic villi demonstrating proliferation of villous capillaries without stromal hypercellularity. ${ }^{12}$ The diagnostic criteria for chorangiosis was given by Altshuler in 1984, as the presence of a minimum of 10 terminal villi, containing more than 10 capillaries per villus in 10 medium power fields in at least three or more random, noninfarcted placental areas. ${ }^{13}$ Suzuki et al. with the use of placental tissue oxygen index values found an association between oxygen saturation of maternal blood in villous spaces and development of chorangiosis. ${ }^{14}$ It has been associated with multiple 
maternal and fetal abnormalities such as preeclampsia, diabetes, hypertension, major congenital anomalies, etc. Various maternal and environmental factors have been implicated in its development such as air pollution, obesity, and smoking. ${ }^{15}$ Chorangiosis affects approximately 5-6\% of pregnancies and usually occurs after 32 weeks of gestation ${ }^{16}$ and fetal morbidity and mortality rate is as high as $42 \% .{ }^{13}$ The differential diagnosis include congestion, placental malperfusion, chorioangioma, and chorioangiomatosis. In placental congestion, the villi show numerically normal vessels, whereas in placental malperfusion, there is severe subtotal placental congestion but diffuse villous capillary hypervascularity is not seen. ${ }^{13}$ Both chorioangioma and chorioangiomatosis show localized proliferation of vascular channels within a single villous covered by trophoblastic tissue. These changes are often seen before 32 weeks of gestation. In chorioangiomatosis, the vessels have a thick wall containing actin-positive smooth muscle cells. ${ }^{12}$ Chorangiosis on the other hand is seen more often after 37 weeks of pregnancy and often leads to fetal distress. In present case also, pregnancy continued till 40 weeks and the patient delivered a growth-restricted baby. Chorangiosis is a diffuse process involving the tips of terminal villi and has numerous closely approximating capillaries with intact basement membrane, an adaptive response to hypoxic stimuli. However, the study suggests that the existence of chorangiosis in many infants with hypoxia still remains unknown because many times placental tissue is not being sent for examination. ${ }^{17}$

Case 3 elaborates likely new clinicopathological entity PMD. It is a rare condition characterized by placentomegaly and multicyclic changes of villi resembling partial molar pregnancy on gross examination. The first two cases were described by Moscoso et al. in $1991 .^{18}$ These cases had elevated maternal serum alpha fetoprotein levels and prenatal USG reveals the appearance of partial hydatidiform mole, however lacking microscopic features of a partial mole like trophoblastic proliferation or stromal trophoblastic inclusions. In the present case, ultrasound revealed cystic changes in the placenta while karyotype of the fetus was normal, causing diagnostic dilemma between partial mole and placenta mesenchymal dysplasia. Later, the histopathology report diagnosed few hydropic changes in the placenta. The true incidence of PMD is still unknown, though there are reports with female preponderance. ${ }^{19}$ Cohen et al. also reported three cases of PMD with aneuploidy, though majority of PMD cases have normal karyotype in contrast to partial mole. Placental mesenchymal dysplasia is sometimes associated with Beckwith-Wiedemann syndrome (BWS); however, in the present case neonate was normal. The differential diagnosis of PMD includes partial molar pregnancy, complete mole with coexisting normal fetus, chorangioma, subchorionic hematoma, and spontaneous abortion with hydropic changes. ${ }^{20}$ Placental mesenchymal dysplasia must be distinguished from partial mole antenatally since later often warrants termination of pregnancy in lieu of complications in the mother or fetus. In the present case, amniocentesis and karyotyping were done to rule out presence of partial mole. The incidence of partial mole with a live fetus also known as Sad fetus syndrome is $0.005-0.01 \%$ of all pregnancies. ${ }^{21}$ Placental mesenchymal dysplasia cases are most often associated with normal fetus with favorable outcome; some cases may have fetal growth restriction, fetal anemia, thrombocytopenia, prematurity, and intrauterine fetal death. ${ }^{22}$ In the present case too, the fetus was growth restricted. This maybe due to diversion of fetal blood to the vascular malformation complex and thrombosis within the malformed vessel. ${ }^{23}$ On USG, PMD appears as cystic lesions of placenta, which later in third trimester present as large vascular spaces; however, in the present case, the spaces were smaller. The placenta of a complete mole with coexisting normal fetus and partial molar pregnancy appears as heterogeneous lesions, with partially solid and cystic areas, ${ }^{24}$ while a chorioangioma appears as a focal lesion and is hypoechoic compared to the rest of the placenta. ${ }^{20}$ The final diagnosis can only be made by histopathology of the placenta. Placental mesenchymal dysplasia is grossly characterized by placentomegaly with diffuse fibromuscular hyperplasia of villi. Microscopic examination of the PMD placenta reveals large stem villi with hydropic swelling and cistern formation interspersed with smaller normal villi. Trophoblastic stromal inclusions and proliferation are absent. ${ }^{25}$ Since both PMD and partial mole can lead to adverse pregnancy outcomes, antenatal surveillance with serial scans and biochemical and genetic evaluation should be done. Though all the sonography and histopathology features were not absolutely same, final diagnosis of the present case was likely PMD by exclusion.

\section{Conclusion}

The placenta is an important guide toward existing genetic, vascular, or structural conditions in the antenatal period, which can adversely affect the fetomaternal outcome. All the three placental conditions were associated with fetal morbidities and two cases were associated with obstetric cholestasis in the current case series. Serial USG assessment of the placenta can tract evolving anomalies. Additional prenatal tests and biomarkers should be conducted to clinch the diagnosis. Gross examination of the placenta should be done in all deliveries and histopathological examination of the placenta should be offered to all women.

\section{References}

1. Gude NM, Roberts CT, Kalionis B, et al. Growth and function of the normal human placenta. Thromb Res 2004;114(5-6):397-407. DOI: 10.1016/j.thromres.2004.06.038.

2. Roescher AM, Timmer A, Erwich JJHM, et al. Placental pathology, perinatal death, neonatal outcome, and neurological development: a systematic review. PLoS ONE 2014;9(2):e89419. DOI: 10.1371/journal. pone.0089419.

3. Hargitai B, Marton T, Cox PM. Best practice no 178. Examination of the human placenta. J Clin Pathol 2004;57(8):785-792. DOI: 10.1136/ jcp.2003.014217.

4. Korteweg FJ, Erwich JJHM, Holm JP, et al. Diverse placental pathologies as the main causes of fetal death. Obstet Gynecol 2009;114(4):809-817. DOI: 10.1097/AOG.0b013e3181b72ebe.

5. Roberts DJ, Oliva E. Clinical significance of placental examination in perinatal medicine. J Matern-Fetal Neonatal Med 2006;19(5):255-264. DOI: 10.1080/14767050600676349.

6. Ahmed N, Kale V, Thakkar H, et al. Sonographic diagnosis of placental teratoma. J Clin Ultrasound JCU 2004;32(2):98-101. DOI: 10.1002/ jcu.10231.

7. Kudva R, Monappa V, Rai L. Placental teratoma: a diagnostic dilemma with fetus acardius amorphous. Indian J Pathol Microbiol 2010;53(2):378-379. DOI: 10.4103/0377-4929.64293.

8. Gillet N, Hustin J, Magritte JP, et al. Placental teratoma: Differential diagnosis with fetal acardia. J Gynecol Obstet Biol Reprod (Paris) 2001;30(8):789-792.

9. Fujikura T, Wellings SR. A teratoma-like mass on the placenta of a malformed infant. Am J Obstet Gynecol 1964;89(6):824-825. DOI: 10.1016/0002-9378(64)90190-5. 
10. Prashanth A, Lavanya R, Girisha KM, et al. Placental teratoma presenting as a lobulated mass behind the neck of fetus: a case report. Case Rep Obstet Gynecol 2012;2012:857230. DOI: 10.1155/2012/857230.

11. Petersen SS, Khangura R, Davydov D, et al. Placental chorangiosis: increased risk for cesarean section [internet]. Case Rep Obstet Gynecol 2017;2017:e5610945. DOI: 10.1155/2017/5610945Available from: https://www.hindawi.com/journals/criog/2017/5610945/.

12. Mathew M, Garg S, Rai L, et al. Placental chorangiosis - a report of two cases with unusual associations and review of literature. Internet J Gynecol Obstet [Internet] 2008;11(2):Available from: http://ispub. com/IJGO/11/2/3660.

13. Altshuler G. A conceptual approach to placental pathology and pregnancy outcome. Semin Diagn Pathol 1993;10(3):204-221.

14. Suzuki K, Itoh H, Kimura S, et al. Chorangiosis and placental oxygenation. Congenit Anom 2009;49(2):71-76. DOI: 10.1111/j.17414520.2009.00226.x.

15. Akbulut M, Sorkun HC, Bir F, et al. Chorangiosis: The potential role of smoking and air pollution. Pathol-Res Pract 2009;205(2):75-81. DOI: 10.1016/j.prp.2008.05.004.

16. Gupta R, Nigam S, Arora P, et al. Clinico-pathological profile of 12 cases of chorangiosis. Arch Gynecol Obstet $2006 ; 274(1): 50-53$. DOI: 10.1007/s00404-005-0076-0.

17. Schwartz D. A guest editorial: chorangiosis and its precursors: underdiagnosed placental indicators of chronic fetal hypoxia. Obstet Gynecol Surv 2001;56(9):523-525. DOI: 10.1097/00006254200109000-00001.
18. Moscoso G, Jauniaux E, Hustin J. Placental vascular anomaly with diffuse mesenchymal stem villous hyperplasia. A new clinicopathological entity? Pathol Res Pract 1991;187(2-3):324-328. DOI: 10.1016/S0344-0338(11)80791-0.

19. Cohen MC, Roper EC, Sebire NJ, et al. Placental mesenchymal dysplasia associated with fetal aneuploidy. Prenat Diagn 2005;25(3):187-192. DOI: $10.1002 /$ pd.1103.

20. Vaisbuch E, Romero R, Kusanovic JP, et al. Three-dimensional sonography of placental mesenchymal dysplasia and its differential diagnosis. J Ultrasound Med Off J Am Inst Ultrasound Med 2009;28(3):359-368. DOI: 10.7863/jum.2009.28.3.359.

21. Agarwal R, Agarwal S, Roy KK, et al. Diploid partial mole with neonatal survival-a case report. Indian J Pathol Microbiol 2005;48(2): 225-227.

22. Pham T, Steele J, Stayboldt C, et al. Placental mesenchymal dysplasia is associated with high rates of intrauterine growth restriction and fetal demise: a report of 11 new cases and a review of the literature. Am J Clin Pathol 2006;126(1):67-78. DOI: 10.1309/RV45HRD53YQ2YFTP.

23. Sander CM. Angiomatous malformation of placental chorionic stem vessels and pseudo-partial molar placentas: report of five cases. Pediatr Pathol 1993;13(5):621-633. DOI: 10.3109/15513819309048250.

24. Mittal D, Anand R, Sisodia N, et al. Placental mesenchymal dysplasia: what every radiologist needs to know. Indian J Radiol Imaging 2017;27(1):62-64. DOI: 10.4103/0971-3026.202949.

25. Ang DC, Rodríguez Urrego PA, Prasad V. Placental mesenchymal dysplasia: a potential misdiagnosed entity. Arch Gynecol Obstet 2009;279(6):937-939. DOI: 10.1007/s00404-008-0812-3. 